\title{
A Physical Model for the Luminosity Function of High\#Redshift Quasars
}

\section{Citation}

Wyithe, J. S. B., and A. Loeb. 2002. "A Physical Model for the Luminosity Function of High\# Redshift Quasars." The Astrophysical Journal 581 (2): 886-94. https://doi.org/10.1086/344249.

\section{Permanent link}

http://nrs.harvard.edu/urn-3:HUL.InstRepos:41393303

\section{Terms of Use}

This article was downloaded from Harvard University's DASH repository, and is made available under the terms and conditions applicable to Other Posted Material, as set forth at http:// nrs.harvard.edu/urn-3:HUL.InstRepos:dash.current.terms-of-use\#LAA

\section{Share Your Story}

The Harvard community has made this article openly available.

Please share how this access benefits you. Submit a story.

\section{Accessibility}




\title{
A PHYSICAL MODEL FOR THE LUMINOSITY FUNCTION OF HIGH-REDSHIFT QUASARS
}

\author{
J. S. B. Wyithe ${ }^{1}$ AND A. Loeb \\ Harvard-Smithsonian Center for Astrophysics, 60 Garden Street, Cambridge, MA 02138; \\ swyithe@cfa.harvard.edu, aloeb@cfa.harvard.edu \\ Received 2002 June 10; accepted 2002 August 19
}

\begin{abstract}
We provide a simple theoretical model for the quasar luminosity function at high redshifts that naturally reproduces the statistical properties of the luminous Sloan Digital Sky Survey (SDSS) quasar sample at redshifts $z \sim 4.3$ and $z \gtrsim 5.7$. Our model is based on the assumptions that quasar emission is triggered by galaxy mergers and that the black hole mass is proportional to a power law in the circular velocity of the host galactic halo, $v_{c}$. We assume that quasars shine at their Eddington luminosity over a time proportional to the mass ratio between the small and final galaxies in the merger. This simple model fits the quasar luminosity function at $z \sim 2-3$, reproduces the normalization and logarithmic slope $(\beta \sim-2.58)$ at $z \sim 4.3$, explains the space density of bright SDSS quasars at $z \sim 6.0$, reproduces the black hole-halo mass relation for dormant black holes in the local universe, and matches the inferred duty cycle of quasar activity $\left(\sim 10^{7} \mathrm{yr}\right)$ in Lyman break galaxies at $z \sim 3$. An acceptable fit to all of these constraints requires $0.7 \lesssim \sigma_{8} \lesssim 1.0$. Based on the derived luminosity function, we predict the resulting gravitational lensing rates for high-redshift quasars. The lens fractions in the SDSS samples are predicted to be $\sim 2 \%$ at $z \sim 4.3$ and $\sim 8 \%$ at $z \gtrsim 5.7$. It is interesting to note that the limiting quasar luminosity in our best-fit relation $L \propto v_{c}^{5} / G$ scales as the binding energy of the host galaxy divided by its dynamical time, implying that feedback is the mechanism that regulates black hole growth in galactic potential wells.

Subject headings: gravitational lensing — quasars: general
\end{abstract}

\section{INTRODUCTION}

While the quasar luminosity function has been studied extensively at redshifts below $z \sim 3$ (e.g., Boyle, Shanks, \& Peterson 1988; Hartwick \& Schade 1990; Pei 1995; Boyle et al. 2000), the Sloan Digital Sky Survey (SDSS; Fukugita et al. 1996; Gunn et al. 1998; York et al. 2001) has, in recent years, substantially increased the number of quasars known at $z \gtrsim 3.5$ (Fan et al. 2001a, 2001b; Schneider et al. 2001). Two samples of very high-redshift SDSS quasars have been presented to date. The first of these is a sample of 39 luminous quasars with redshifts in the range $3.6<z<5.0$ and a median of 4.3. The second sample consists of four quasars at $z \gtrsim 5.7$, including the quasar with the highest known redshift $(z=6.28)$. These samples begin to sketch out the luminosity function of quasars at $z \gtrsim 4$ and are very important for studies of the ionizing background radiation and of quasar evolution around the epoch of reionization (Haiman \& Loeb 1998). Although the logarithmic slope of the luminosity function for bright quasars admits a universal value of $\sim-3.5$ at redshifts $z \lesssim 3$, Fan et al. (2001a) find a shallower logarithmic slope of $\sim-2.5$ for their luminous quasars at $z \gtrsim 3.5$. At first sight, this is a puzzling result because the exponential tail of the Press-Schechter (1974) mass function describing the space density of the massive host galaxies in which bright quasars are thought to reside becomes progressively steeper with increasing redshift.

There are several published models of varying complexity to describe the observed evolution of the quasar luminosity function within hierarchical structure formation theory (e.g., Efstathiou \& Rees 1988; Small \& Blandford 1992; Haehnelt \& Rees 1993; Haiman \& Loeb 1998; Haehnelt

${ }^{1}$ Hubble Fellow.
Natarajan, \& Rees 1998; Kauffmann \& Haehnelt 2000). In these models, the luminosities and lifetimes of the quasars are governed by the black hole mass and the supply of cold accreting gas. The abundance and evolution of the supermassive black holes assumed to power the quasars are linked to the evolution of the mass-function of galactic halos. The density of bright quasars is observed to decline rapidly with redshift below $z \sim 2$ (Boyle, Shanks, \& Peterson 1988; Hartwick \& Schade 1990). This decline has been explained (Kauffman \& Haehnelt 2000; see also Cavaliere \& Vittorini 2000) in terms of decreases in the merger rate and the availability of cold gas to fuel the black holes. In addition, the density of bright quasars is also observed to decline beyond a redshift of $z \sim 2$ (Warren, Hewett, \& Osmer 1994; Schmidt, Schneider, \& Gunn 1995; Kennefick, Djorgovski, \& de Carvalho 1995). Under the assumption that quasar activity is related to the formation rate of halos, model luminosity functions have been constructed that successfully describe this decline and the shape of the luminosity function between $z \sim 2$ and $z \sim 3.5$ (Efstathiou \& Rees 1988; Haehnelt \& Rees 1993; Haiman \& Loeb 1998; Haehnelt, Natarajan, \& Rees 1999; Haiman, Madau, \& Loeb 1999). These models predict the luminosity function at still higher redshifts. With the recent influx of results on the quasar luminosity at redshifts above $z \sim 4$, it is interesting to revisit the question of modeling the evolution of the quasar luminosity function at high redshifts. In this paper, we demonstrate that a very simple model can explain the new results on the high-redshift quasar luminosity function.

Our model for the evolution of the quasar luminosity function is based on the Press-Schechter (1974) mass function and halo merger rates computed with the excursion set formalism (Bond et al. 1991; Lacy \& Cole 1993). We show that this model can reproduce all of the known properties of the luminosity function above $z \sim 2$. We begin in $\S 2$ by 
describing an approximate version of our model (Haiman \& Loeb 1998, hereafter HL98), followed by the full discussion based on the halo merger rates. In $\S 3$ and $\S 4$, we discuss the data on the quasar luminosity function and its comparison with our model. Finally, we discuss the implications of our model for gravitational lensing in high-redshift samples of quasars in $\S 5$ and present our main conclusions in $\S 6$. Throughout the paper, we assume density parameters of $\Omega_{m}=0.35$ in matter, $\Omega_{\Lambda}=0.65$ in a cosmological constant, and a Hubble constant of $H_{0}=65 \mathrm{~km} \mathrm{~s}^{-1} \mathrm{Mpc}^{-1}$. For calculations of the Press-Schechter (1974) mass function, we assume a primordial power-spectrum with $n=1$ and the fitting formula to the exact transfer function of Bardeen et al. (1986). Unless otherwise noted, we take $\sigma_{8}=0.87$.

\section{THEORETICAL LUMINOSITY FUNCTIONS}

We discuss two models for the high-redshift quasar luminosity function that are based on the Press-Schechter (1974) mass function of dark matter halos (including the modification of Sheth \& Tormen 1999) and the halo merger rates from the excursion set formalism of Bond et al. (1991) and Lacy \& Cole (1993). We begin with the approximate model of HL98, which is based on the halo formation rate. We then describe a more complete model based on halo merger rates. We will demonstrate in $\S 4.1$ that only this latter model reproduces all the known features of the quasar luminosity function above $z \sim 2$ as well as the local black holehost halo mass relation and new estimates of the quasar duty cycle.

\subsection{An Approximate Model for the Quasar Luminosity Function Based on the Halo Formation Rate}

HL98 discussed an approximate model for the highredshift quasar luminosity function by supposing that quasars are associated with newly formed halos. Their model assumes that each galactic halo hosts a black hole with a mass $\left(M_{\mathrm{bh}}\right)$ that is a constant fraction $(\epsilon)$ of the host halo mass $\left(M_{\text {halo }}\right)$ and that the black hole shines at its Eddington rate with a universal light curve, $f(t)$. HL98 used the derivative of the Press-Schechter mass function to approximate the formation rate of halos, arguing that mergers are rare at high redshift so that the negative contribution to the derivative is negligible. In this model, the quasar luminosity as a function of time is

$$
L_{B}(t)=M_{\text {bh }} f(t)=\epsilon M_{\text {halo }} f(t) \text { for } M_{\text {halo }}>M_{\text {min }},
$$

where $M_{\min } \sim 10^{8} M_{\odot}[(1+z) / 10]^{-3 / 2}$ is the minimum halo mass inside which a black hole can form. This lower mass limit corresponds to the virial temperature below which atomic cooling is not effective in allowing the gas to sink to the center (Barkana \& Loeb 2001). Following HL98, we write the resulting quasar luminosity function, defined as the comoving number density of quasars having rest-frame $B$-band luminosities between $L_{B}$ and $L_{B}+\Delta L_{B}$ and redshifts between $z$ and $z+\Delta z$, as

$$
\begin{aligned}
\Psi_{\mathrm{HL}}\left(L_{B}, z\right)= & \int_{z}^{\infty} d z^{\prime} \int_{\epsilon \mathrm{M}_{\min }}^{\infty} d M_{\mathrm{bh}} \frac{d^{2} n_{\mathrm{bh}}}{d M_{\mathrm{bh}} d z^{\prime}} \\
& \times \delta\left[L_{B}-M_{\mathrm{bh}} f\left(t_{\mathrm{z}}-t^{\prime}\right)\right],
\end{aligned}
$$

where $t_{z}$ and $t^{\prime}$ are the time at redshifts $z$ and $z^{\prime}$, and $d^{2} n_{\mathrm{bh}} / d M_{\mathrm{bh}} d z^{\prime}$ is the change in the comoving number density of black holes between $z^{\prime}$ and $z^{\prime}+d z^{\prime}$. Integrating over $M_{\text {bh }}$, we get

$$
\begin{aligned}
\Psi_{\mathrm{HL}}\left(L_{B}, z\right)= & \int_{0}^{a=1 /(1+z)} d a \frac{d z}{d a} \frac{1}{f\left(t_{\mathrm{z}}-t\right)} \\
& \times\left|\frac{d^{2} n_{\mathrm{bh}}}{d M_{\mathrm{bh}} d z}\right|_{M_{\mathrm{bh}}=L_{B} /\left[f\left(t_{z}-t\right)\right]},
\end{aligned}
$$

where $a=(1+z)^{-1}$ is the scale factor at a redshift $z$. HL98 used an exponential with an $e$-folding time $t_{\mathrm{dc} .0}$ for the universal light curve $f$. We find that the equivalent result for $\Psi_{\mathrm{HL}}\left(L_{B}, z\right)$ is obtained more simply by assuming a step function for $f$, namely

$$
f(t)=\frac{L_{\mathrm{Edd}, B}}{M_{\mathrm{bh}}} \Theta\left(t-t_{\mathrm{dc}, 0}\right),
$$

where $L_{\mathrm{Edd}, B}=5.7 \times 10^{3}\left(M_{\mathrm{bh}} / M_{\odot}\right)$ is the Eddington luminosity in $B$-band solar luminosity units for the median quasar spectrum (Elvis et al. 1994) and $\Theta(t)$ is the Heaviside step function. We then find

$$
\begin{aligned}
\Psi_{\mathrm{HL}}\left(L_{B}, z\right)= & \int_{a_{\min }=1 /\left(1+z_{\mathrm{form}}\right)}^{a=1 /(1+z)} d a \frac{d z}{d a} \frac{M_{\mathrm{bh}}}{L_{\mathrm{Edd}, B}} \\
& \times\left|\frac{d^{2} n_{\mathrm{bh}}}{d M_{\mathrm{bh}} d z}\right|_{M_{\mathrm{bh}}=L_{B} /\left[f\left(t_{z}-t\right)\right]},
\end{aligned}
$$

given the formation redshift for the halo, $z_{\text {form }}$. Assuming that $t_{\mathrm{dc}, 0} \ll H^{-1}(z),\left(a-a_{\min }\right) \approx(d a / d t) t_{\mathrm{dc}, 0}$, and relating the space density of black holes to the Press-Schechter (1974) mass function through

$$
\frac{d^{2} n_{\mathrm{bh}}}{d M_{\mathrm{bh}} d z}=\frac{1}{\epsilon} \frac{d^{2} n_{\mathrm{ps}}}{d M_{\mathrm{halo}} d z},
$$

we obtain the following simple expression for the quasar luminosity function:

$$
\begin{aligned}
\Psi_{\mathrm{HL}}\left(L_{B}, z\right) \approx & \frac{t_{\mathrm{dc}, 0}}{H_{0}^{-1}} \sqrt{\frac{\Omega_{m}}{a^{5}}+\frac{\Omega_{\Lambda}}{a^{2}}} \frac{1}{5.7 \times 10^{3}} \\
& \times \frac{1}{\epsilon}\left|\frac{d^{2} n_{\mathrm{ps}}}{d M_{\text {halo }} d z}\right|_{M_{\text {halo }}=L_{B} /\left(5.7 \times 10^{3} \epsilon\right)} .
\end{aligned}
$$

We will compare this model to observations in $\S 4.2$.

\subsection{A Model for the Quasar Luminosity Function Based on the Halo Merger Rate}

The HL98 model associates quasar activity with halo formation. The assumption is that the halo merger rate is much lower than the halo formation rate at high redshift. However, at low redshifts, the formation rate becomes smaller than the merger rate, and the derivative of the PressSchechter mass function on which the model is based no longer approximates the halo formation rate. Quasar activity at low redshift can be explained in relation to halo merger activity (Carlberg 1990; Kauffmann \& Haehnelt 2000). In this section, we discuss a simple model that associates quasar activity at high redshift with the merger rate of halos.

The merger rate of halos was computed by Lacy \& Cole (1993) based on the excursion set formalism of Bond et al. (1991). We compute the number of halos having masses between $\Delta M_{\text {halo }}$ and $\Delta M_{\text {halo }}+d \Delta M_{\text {halo }}$ that accrete onto a 
halo of mass $M_{\text {halo }}-\Delta M_{\text {halo }}$ per unit time $\mid\left(d^{2} N_{\text {merge }}\right) /$ $\left.\left(d \Delta M_{\text {halo }} d t\right)\right|_{M_{\text {bal }}-\Delta M_{\text {ban }}}$. Thus, the number of merger events involving the accretion of a halo of mass $\Delta M_{\text {halo }}$ by a halo of mass $M_{\text {halo }}-\Delta M_{\text {halo }}$ per unit time per comoving volume at a redshift $z$ is given by the product

$$
\left|\frac{d n_{\mathrm{ps}}}{d M}\right|_{M=M_{\text {halo }}-\Delta M_{\text {halo }}} \times\left|\frac{d^{2} N_{\text {merge }}}{d \Delta M_{\text {halo }} d t}\right|_{M_{\text {halo }}-\Delta M_{\text {halo }}} \cdot
$$

We assume that the mass of the central black hole scales as a power law with the circular velocity $\left(v_{\mathrm{c}}\right)$ of the halo,

$$
M_{\mathrm{bh}} \propto v_{c}^{\gamma} .
$$

The circular velocity of a halo of mass $M_{\text {halo }}$ at redshift $(z)$ can be written as (Barkana \& Loeb 2001)

$$
\begin{aligned}
v_{c}= & 159.4\left(\frac{M_{\text {halo }}}{10^{12} h^{-1} M_{\odot}}\right)^{1 / 3}\left(\frac{\Omega_{m}}{\Omega_{m}^{z}} \frac{\Delta_{c}}{18 \pi^{2}}\right)^{1 / 6} \\
& \times(1+z)^{1 / 2} \mathrm{~km} \mathrm{~s}^{-1},
\end{aligned}
$$

where $\quad h=\left(H_{0} / 100 \mathrm{~km} \mathrm{~s}^{-1} \mathrm{Mpc}^{-1}\right), \quad \Delta_{\mathrm{c}}=18 \pi^{2}+82 d$ $-39 d^{2}$ is the final overdensity relative to the critical density at redshift $z$, and $d \equiv 1-\Omega_{m}^{z}$ with $\Omega_{m}^{z}=$ $\left[\Omega_{m}(1+z)^{3}\right] /\left[\Omega_{m}(1+z)^{3}+\Omega_{\Lambda}\right]$. We can therefore write

$$
\begin{aligned}
\frac{M_{\mathrm{bh}}}{M_{\text {halo }}}= & \epsilon=\epsilon_{0}\left(\frac{M_{\text {halo }}}{10^{12} M_{\odot}}\right)^{(\gamma / 3)-1}\left(\frac{\Omega_{m}}{\Omega_{m}^{z}} \frac{\Delta_{c}}{18 \pi^{2}}\right)^{\gamma / 6} \\
& \times h^{\gamma / 3}(1+z)^{\gamma / 2},
\end{aligned}
$$

and fit for two free parameters, $\epsilon_{0}$ and $\gamma$, that describe the evolution and slope of the black hole-halo mass relation. We assume that the black holes coalesce upon halo merger (Kauffmann \& Haehnelt 2000) and find the number of black holes of mass between $\Delta M_{\mathrm{bh}}$ and $\Delta M_{\mathrm{bh}}+d \Delta M_{\mathrm{bh}}$ that merge with black holes of mass $M_{\mathrm{bh}}-\Delta M_{\mathrm{bh}}$ per unit time:

$$
\left|\frac{d^{2} N_{\text {merge }}}{d \Delta M_{\mathrm{bh}} d t}\right|_{M_{\mathrm{bh}}-\Delta M_{\mathrm{bh}}}=\frac{3}{\gamma \epsilon}\left|\frac{d^{2} N_{\text {merge }}}{d \Delta M_{\text {halo }} d t}\right|_{M_{\text {halo }}-\Delta M_{\text {halo }}} .
$$

A linear relation $(\gamma=3)$ between black hole mass and halo mass is a natural consequence of black hole growth that is dominated by coalescence with no gas accretion (Haehnelt et al. 1998). Values of $\gamma>3$ result from significant gas accretion during the active quasar phase as deduced by $\mathrm{Yu}$ $\&$ Tremaine (2002). We assume that after a merger, a fraction of the cold gas from the accreted halo is driven onto the central black hole of mass $M_{\mathrm{bh}}$ (Mihos \& Hernquist 1994; Hernquist \& Mihos 1995). If the quasar shines at the Eddington rate of the black hole in the merger product (see, e.g., Yu \& Tremaine 2002), then the cold gas from the small accreted halo (which makes up the new fuel reservoir) will run out in a time approximately proportional to

$$
\frac{\Delta M_{\text {baryon }}}{M_{\text {halo }}}=\frac{\Delta M_{\text {halo }}}{M_{\text {halo }}} \frac{\Delta M_{\text {baryon }}}{\Delta M_{\text {halo }}},
$$

where $\Delta M_{\text {baryon }}$ is the mass in baryons within the accreted halo. ${ }^{2}$ Thus, we postulate that after a merger, the quasar shines at the Eddington rate corresponding to the merger

${ }^{2}$ Eq. (13) can be derived as a Taylor expansion for minor mergers. For simplicity, we apply it to all mergers. product for a time proportional to both the ratio between the masses of the accreted and initial halo and the baryon fraction of the accreted halo.

In order to examine whether the baryon mass fraction in the accreted halo would be affected by the photoionization heating of the intergalactic medium (IGM) after reionization, we have solved the linear growth factors $D_{\mathrm{dm}}$ and $D_{b}$ for the dark matter and baryons on a spatial comoving scale $R=\left(3 \Delta M_{\text {halo }} / 4 \pi \rho_{m}\right)^{1 / 3}$, where $\rho_{m}$ is the average matter density of the universe today. These growth factors obey the following set of coupled differential equations for the dark matter and baryon overdensities $\delta_{\mathrm{dm}}$ and $\delta_{b}$ in the linear regime (e.g., Barkana \& Loeb 2001):

$$
\begin{aligned}
\ddot{\delta}_{\mathrm{dm}}+2 H \dot{\delta}_{\mathrm{dm}}= & \frac{3}{2} H^{2}\left[\Omega_{b}(z) \delta_{b}+\Omega_{\mathrm{dm}}(z) \delta_{\mathrm{dm}}\right], \\
\ddot{\delta}_{b}+2 H \dot{\delta_{b}}= & \frac{3}{2} H^{2}\left\{\left[\Omega_{b}(z) \delta_{b}+\Omega_{\mathrm{dm}}(z) \delta_{\mathrm{dm}}\right]\right\}-\frac{k_{\mathrm{B}} T_{i}}{\mu m_{p}}\left(\frac{k}{a}\right)^{2} \\
& \times\left(\frac{a_{i}}{a}\right)^{1+\beta}\left[\delta_{b}+\frac{2}{3} \beta\left(\delta_{b}-\delta_{b, i}\right)\right] .
\end{aligned}
$$

Here $T$ is the gas temperature, $\mu$ the mean molecular weight ( $\sim 0.69$ for ionized IGM), $k_{\mathrm{B}}$ is Boltzmann's constant, and $k$ is the comoving wave number for the mode of interest. The subscripts $i$ refer to quantities at an initial reference time and $\beta=0$ or 1 for adiabatic or isothermal evolution. We assume reionization at $z_{\text {reion }}=7$. Before reionization, we assume $T_{i}=0$ : the baryon and dark matter overdensities undergo the same evolution during this period. Following reionization, the IGM is assumed to be heated to $10^{4} \mathrm{~K}$ and to evolve isothermally $(\beta=1)$. The evolution of $D_{b}$ is described by the superposition of solutions to equation (14) weighted by the Fourier transform of the spatial top hat window function of width $R$ (Medvigy \& Loeb 2001). Figure 1 shows the evolution of $D_{b}$ with redshift. The curves shown correspond (from bottom to top) to halo masses of $M_{\text {halo }}=10^{6}, 10^{7}, 10^{8}, 10^{9}, 10^{10}, 10^{11}, 10^{12}, 10^{13}$,

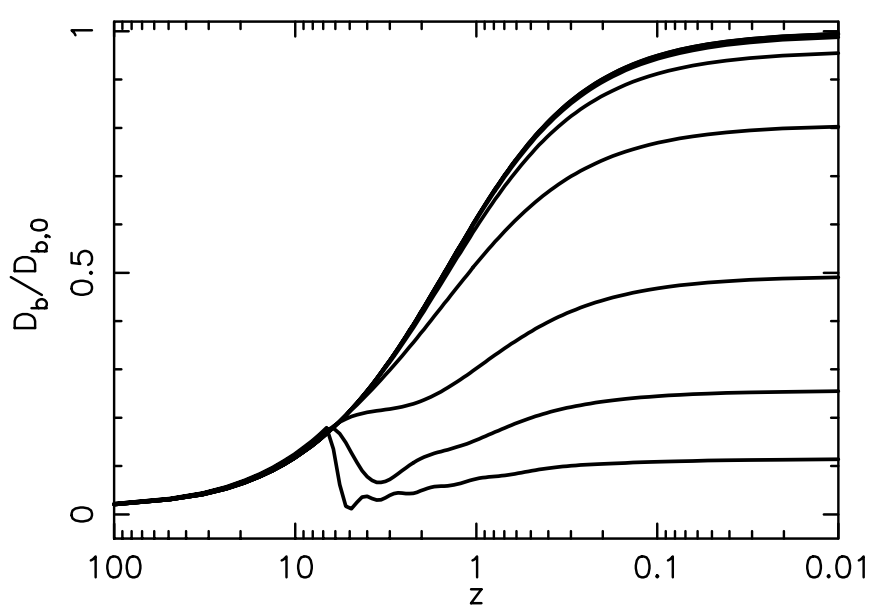

FIG. 1.-Growth factor $D_{b}$ (normalized to the present-day $D_{b, 0}$ ) for baryons associated with dark matter halos of mass $M_{\text {halo }}=10^{6}, 10^{7}, 10^{8}$, $10^{9}, 10^{10}, 10^{11}, 10^{12}, 10^{13}$, and $10^{14} M_{\odot}$ (bottom to top). The downturn of the growth factor for small halos corresponds to the reionization epoch at $z_{\text {reion }} \sim 7.0$. 
and $10^{14} M_{\odot}$. The dark matter growth factor corresponds to the upper envelope in this figure. Prior to reionization $D_{b}$ follows $D_{\mathrm{dm}}$ for all halo masses. Reinonizaton heats the IGM and eliminates gas accretion onto small halos. The baryon overdensity in halos larger than $10^{10} M_{\odot}$ is unaffected by reionization. Thus, we find that the effect of reionization on the luminosity function is apparent only for quasars fainter than $\sim 10^{11} L_{\odot}$, which is well below the detection threshold of existing quasar surveys at $z \gg 1$, such as SDSS. We therefore assume a fixed baryon mass fraction in objects relevant for the luminosity function of bright quasars at high redshifts.

The quasar light curve and luminosity function may therefore be written as

$$
f(t)=\frac{L_{\mathrm{Edd}, B}}{M_{\mathrm{bh}}} \Theta\left(t-\frac{\Delta M_{\text {halo }}}{M_{\text {halo }}} t_{\mathrm{dc}, 0}\right),
$$

and

$$
\begin{aligned}
\Psi\left(L_{B}, z\right)= & \int_{\epsilon M_{\min }}^{\infty} d M_{\mathrm{bh}} \int_{0}^{0.5 M_{\mathrm{bh}}} d \Delta M_{\mathrm{bh}} \int_{z}^{\infty} \\
& \times d z^{\prime}\left|\frac{d n_{\mathrm{bh}}}{d M}\right|_{M=M_{\mathrm{bh}}-\Delta M_{\mathrm{bh}}}\left|\frac{d^{2} N_{\text {merge }}}{d \Delta M_{\mathrm{bh}} d t^{\prime}}\right|_{M_{\mathrm{bh}}-\Delta M_{\mathrm{bh}}} \\
& \times \frac{d t^{\prime}}{d z^{\prime}} \delta\left[L_{B}-M_{\mathrm{bh}} f\left(t_{z}-t^{\prime}\right)\right],
\end{aligned}
$$

in analogy with equations (4) and (5), respectively. Integrating over $M_{\mathrm{bh}}$, we find

$$
\begin{aligned}
\Psi\left(L_{B}, z\right)= & \int_{0}^{0.5 M_{\text {halo }}} d \Delta M_{\text {halo }} \int_{\mathrm{a}_{\min }=1 /\left(1+z_{\text {form }}\right)}^{a=1 /(1+z)} \\
& \times d a \frac{d z}{d a}\left|\frac{d n_{\mathrm{ps}}}{d M}\right|_{M=M_{\text {halo }}-\Delta M_{\text {halo }}} \\
& \times\left|\frac{d^{2} N_{\text {merge }}}{d \Delta M_{\text {halo }} d t}\right|_{M_{\text {halo }}-\Delta M_{\text {halo }}} \frac{3}{\gamma \epsilon} \frac{d t}{d z} \frac{M_{\mathrm{bh}}}{L_{\mathrm{Edd}, B}} .
\end{aligned}
$$

Assuming $\quad t_{\mathrm{dc}, 0} \ll H^{-1}(z)$, we then obtain $a-a_{\min }=(d a / d t) t_{\mathrm{dc}, 0}\left(\Delta M_{\text {halo }} / M_{\text {halo }}\right)$, yielding the luminosity function

$$
\begin{aligned}
\Psi\left(L_{B}, z\right)= & \int_{0}^{0.5 M_{\text {halo }}} d \Delta M_{\text {halo }} \frac{3}{\gamma \epsilon} \frac{t_{\mathrm{dc}, 0}}{5.7 \times 10^{3}} \frac{\Delta M_{\text {halo }}}{M_{\text {halo }}} \\
& \times\left|\frac{d n_{\mathrm{ps}}}{d M}\right|_{M=M_{\text {halo }}-\Delta M_{\text {halo }}}\left|\frac{d^{2} N_{\text {merge }}}{d \Delta M_{\text {halo }} d t}\right|_{M_{\text {halo }}-\Delta M_{\text {halo }}},
\end{aligned}
$$

where $M_{\text {halo }}=L_{B} / 5.7 \times 10^{3} \epsilon$. The value of $t_{\mathrm{dc}, 0}$ can be related to the duty cycle of quasars $t_{\mathrm{dc}}$ through consideration of the number of mergers during a Hubble time $H^{-1}(z)$,

$$
\begin{aligned}
t_{\mathrm{dc}}\left(L_{B}, z\right)= & t_{\mathrm{dc}, 0} \int_{0}^{M_{\text {halo }}=\left(0.5 L_{B}\right) /\left(5.7 \times 10^{3} \epsilon\right)} d \Delta M_{\text {halo }} \\
& \times \frac{\Delta M_{\text {halo }}}{M_{\text {halo }}} H^{-1}(z)\left|\frac{d^{2} N_{\text {merge }}}{d \Delta M_{\text {halo }} d t}\right|_{M_{\text {halo }}-\Delta M_{\text {halo }}},
\end{aligned}
$$

We will compare the luminosity function predicted by this model with observations in $\S 4.1$.

\section{OBSERVED LUMINOSITY FUNCTION}

The standard double power-law luminosity function (Boyle, Shanks, \& Peterson 1988; Pei 1995)

$$
\Psi\left(L_{B}, z\right)=\frac{\Psi_{*} / L_{*}(z)}{\left[L_{B} / L_{*}(z)\right]^{\beta_{1}}+\left[L_{B} / L_{*}(z)\right]^{\beta_{h}}}
$$

provides a good representation of the observed quasar luminosity function at redshifts $z \lesssim 3$. At the faint end of the luminosity function, the slope is $\beta_{1}=1.58$, while at the bright end $\beta_{h}=3.43$ (Boyle et al. 2000). Moreover, all dependence on redshift (at $z \lesssim 2$ ) is in the break luminosity $L_{*}$, indicating pure luminosity evolution. At low redshift, the break luminosity evolves approximately as a power law in redshift, and the space density of bright quasars increases with redshift. However, surveys at higher redshift show a decline in the space density of bright quasars beyond $z \sim 3$ (Warren, Hewett, \& Osmer 1994; Schmidt, Schneider, \& Gunn 1995; Kennefick, Djorgovski, \& de Carvalho 1995).

While quasar evolution below $z \sim 2-3$ is described by pure luminosity evolution, Fan et al. (2001a) found from their sample of SDSS quasars at $z \sim 4.3$ that the slope at the bright end of the luminosity function has evolved from the $z \lesssim 3$ value of $\beta_{h} \sim-3.5$ to $\beta_{h} \sim-2.5$. This result is supported by the analysis of Schmidt, Schneider, \& Gunn (1995). Fan et al. (2001a) also find an evolution of space density with redshift for bright quasars of $\Psi \propto 10^{-0.5 z}$ between $z \sim 3.5$ and $z \sim 5$. The space density of bright quasars measured by Fan et al. (2001b) at $z \sim 6$ agrees with the extrapolation of this evolution.

We seek to model the observed features of the luminosity function at redshifts higher than the peak in quasar evolution at $z \sim 2$. In the following section, we will discuss both our model (§ 2.2) and the HL98 model in light of the recently acquired data on quasars at high redshifts.

\section{COMPARISON OF OBSERVED AND MODEL LUMINOSITY FUNCTIONS}

In this section, we compute model luminosity functions at different redshifts and compare them with the observed quasar luminosity function. Figure 2 shows the observed luminosity function at $z=0.1,1.0,2.3,3.0,4.0, z \sim 4.3$, and $z \sim 6.0$. Below $z \sim 4.3$, the data points shown are from the summary by Pei (1995; based on the compilations of Hartwick \& Schade 1990). The gray lines at redshifts below 2 are fits to the empirical $2 \mathrm{dF}$ luminosity function of Boyle et al. (2000; we plot their $q_{0}=0.5$ polynomial evolution model corrected for cosmology). We also show the best-fit empirical luminosity function at $z \sim 4.3$ from Fan et al. (2001a) with a vertical bar to denote the quoted error in the normalization. At $z \sim 6$, the space density was inferred from Fan et al. (2001b), and the error bar includes uncertainty in the spectral index. Comparisons of this data with the halo merger rate model (§ 4.1) and the HL98 model (§ 4.2) are presented below.

\subsection{The Merger Rate Model}

Luminosity functions calculated from the halo merger rate model described in $\S 2.2$ are plotted as the solid lines in Figure 2 at redshifts $z=0.1,1.0,2.3,3.0,4.0, z \sim 4.3$ and $z \sim 6.0$. In addition, the lower right panel shows predicted luminosity functions at $z=8.0$ and $z=10.0$. Values of 

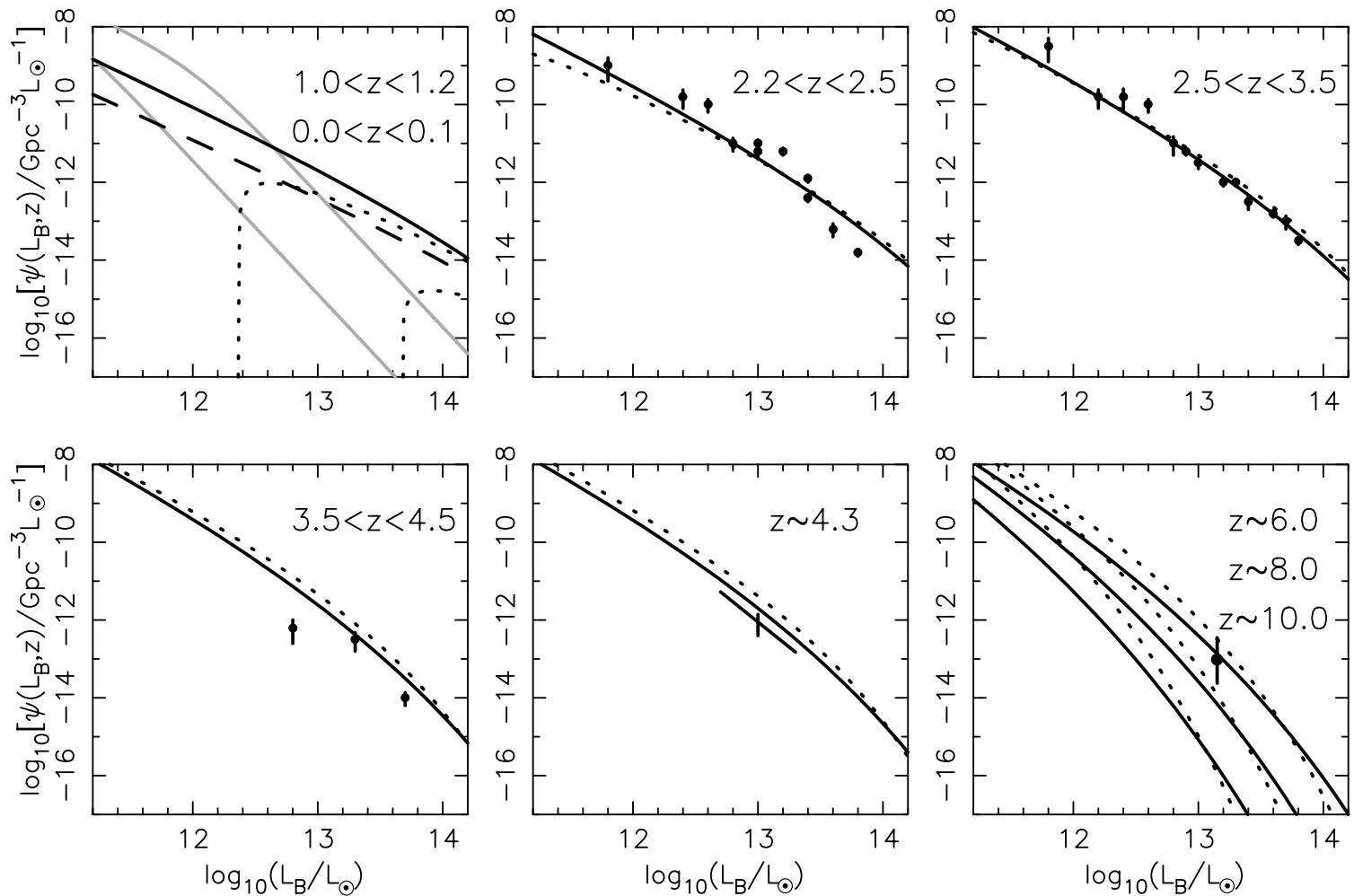

Fig. 2.-Comparison of the observed and model rest-frame $B$-band luminosity functions. The data points at $z \lessgtr 4$ are summarized in Pei (1995). The gray lines in the lowest redshift panel are fits to the empirical 2dF luminosity function (Boyle et al. 2000). At $z \sim 4.3$ and $z \sim 6.0$, the data are from Fan et al. (2001a, 2001b). Panel $z \sim 4.3$ : Best-fit slope of -2.58 measured by Fan et al. (2001a; diagonal line) and the quoted uncertainty in the normalization (vertical bar). In the lowest-redshift panel, the dashed line represents the merger model luminosity function at $z \sim 0.1$. Dotted lines: HL98 model $\left(\epsilon=10^{-3.1}\right.$, $\left.t_{\mathrm{dc}, 0}=10^{5.0} \mathrm{yr}\right)$. Solid lines: Merger model described in this paper $\left(\epsilon_{0}=10^{-5.4}, \gamma=5, t_{\mathrm{dc}, 0}=10^{6.3} \mathrm{yr}\right)$.

$\epsilon_{0}=10^{-5.4}, \gamma=5$, and $t_{\mathrm{dc}, 0}=10^{6.3}$ yr yield a good fit to the data at all $z \gtrsim 2$. The parameters in the black hole-halo mass relation bear a striking resemblance to those in the relation found by Ferrarese (2002) for the local universe, which, in our notation, correspond to $\epsilon_{0}=10^{-5.1}$ and $\gamma=4.71$.

The value of $t_{\mathrm{dc}, 0}$ is related to the duty cycle for different quasar luminosities and redshifts through equation (19). The duty cycle is plotted in Figure 3. For a given halo mass, $t_{\mathrm{dc}}$ is longer at higher redshift. Steidel et al. (2002) inferred the lifetime of bright quasar activity in a large statistical sample of Lyman break galaxies at $z \sim 3$ to be $\sim 10^{7} \mathrm{yr}$, which is surprisingly close to our expected value of $t_{\mathrm{dc}}=10^{6.9} \mathrm{yr}$. Above $z \sim 3$, we find $t_{\mathrm{dc}} \sim 10^{7} \mathrm{yr}$, comparable to the value inferred by comparing the local black hole density with the quasar luminosity function (e.g., Yu \& Tremaine 2002) or by comparing the clustering properties of the $2 \mathrm{dF}$ quasars with a model combining the formation and evolution of quasars with $N$-body simulations (Kauffmann $\&$ Haehnelt 2002). Note that while $t_{\mathrm{dc}}$ becomes a substantial fraction of the age of the universe at the highest redshifts considered, individual episodes of quasar activity are shorter than $t_{\mathrm{dc}, 0}$, which is an order of magnitude smaller.

Two results from the comparison between model and data stand out. First, the halo merger model reproduces the shallow slope for the $z \sim 4.3$ quasars observed by Fan et al. (2001a) and the space density of quasars at $z \sim 6.0$ measured by Fan et al. (2001b). A detailed comparison between the data and the model at $z \sim 4.3$ is shown in Figure 4 . The left-hand panel shows the luminosity function in the region measured by Fan et al. (2001a). The straight line with the error bar represents the empirical luminosity function (bestfit slope of $\beta=-2.58$ ) and the uncertainty in its normalization. The upper solid line is the luminosity function of the merger rate model. The theoretical luminosity function slope is consistent with that of the observed quasars over the measured luminosity range. This is shown more clearly on the right hand panel of Figure 4, where the derivative of

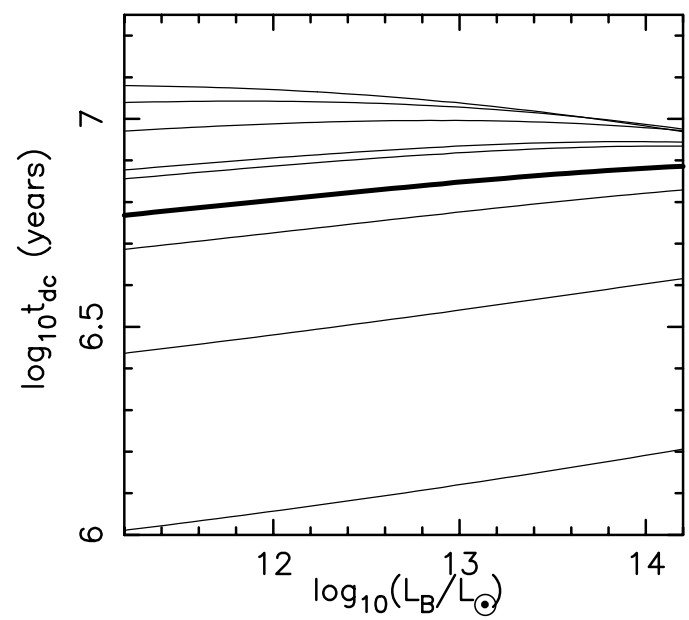

FIG. 3. - Duty cycle time $\left(t_{\mathrm{dc}}\right)$ of quasars with luminosity $L_{B}$ calculated from eq. (19) at redshifts $z=0.1,1.0,2.3,3.0,4.0,4.3,6.0,8.0$, and 10.0 (bottom to top). Thick line: Value at $z=3.0$, which should be compared with $\sim 10^{7}$ yr estimated by Steidel et al. (2002). The duty cycle was calculated using our best-fit value of $t_{\mathrm{dc}, 0}=10^{6.3} \mathrm{yr}$ per merger. 

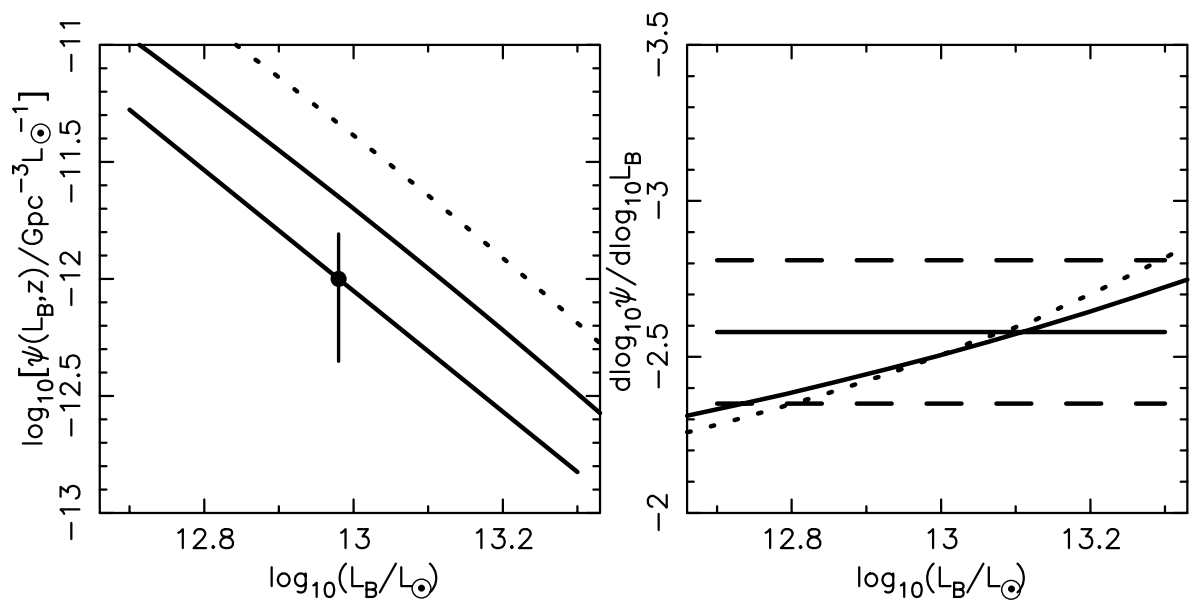

FIG. 4.-Detailed comparison of the observed rest-frame $B$-band quasar luminosity function with the models at $z \sim 4.3$. Left panel: Luminosity function in the region measured by Fan et al. (2001a). Straight line with the error bar: Empirical luminosity function (best-fit slope of $\beta=-2.58$ ) and the quoted uncertainty in the normalization. Upper solid and dotted lines: Luminosity functions for the halo merger model $\left(\epsilon_{0}=10^{-5.4}, \gamma=5, t_{\mathrm{dc} .0}=10^{6.3} \mathrm{yr}\right)$ and for the HL98 model $\left(\epsilon=10^{-3.1}, t_{\mathrm{dc}, 0}=10^{5.0} \mathrm{yr}\right)$, respectively. Right panel: Logarithmic derivatives of the model luminosity functions. Solid and dotted lines: Our model and the HL98 model, respectively. The empirical best-fit slope (solid line) and the slopes $\pm 1 \sigma_{\beta}= \pm 0.23$ away (dashed line) are shown for comparison.

the model luminosity function is shown in comparison with the empirical best-fit slope (solid line) and the slope $\pm 1 \sigma_{\beta}$ away (dashed lines). The model is consistent within the quoted uncertainty over the whole measured luminosity range. Our model luminosity function is consistent with the observed lower limit on the slope at $z=6.0$, which Fan et al. (2000b) quote as $\beta_{h}<3.9$ (95\%). However, the gravitational lensing rate may be very high in this sample (Wyithe \& Loeb 2002a, 2002b). While the average slope of the luminosity function cannot be changed significantly by lensing, we might easily be misled by the magnification of one or two quasars in the limited current sample of four. Furthermore, lensing might increase the apparent space density of quasars by a factor of up to 2 (Wyithe \& Loeb 2002b). The predicted luminosity functions show that the slope of the bright end becomes significantly steeper at redshifts higher than $z \sim 6$, as expected from the shape of the Press-Schechter (1974) mass function.

The fit to the luminosity function discussed above has $\gamma=5$. However, we find some degeneracy between $\gamma$ and $\epsilon_{0}$. An equally good fit to the luminosity function can be obtained for $\gamma=4$ and $\epsilon_{0}=10^{-4.5}$. However, this value of $\epsilon_{\mathrm{o}}$ is nearly an order of magnitude larger than observed (Ferrarese 2002), and the implied duty cycle is nearly an order of magnitude too small (Yu \& Tremaine 2002; Steidel et al. 2002). On the other hand, the observed values of $\gamma=4.71$ and $\epsilon=10^{-5.1}$ (Ferrarese 2002) also provide an excellent fit and a duty cycle consistent with $10^{7} \mathrm{yr}$. Hence, the complete set of observational constraints favors a larger value of $\gamma$. The degeneracy between the ratio of black hole to dark matter halo mass and the quasar lifetime was previously pointed out by Haenhelt, Natarayan, \& Rees (1998). Using a model similar to that employed by HL98, they found that a quasar duty cycle of $\sim 10^{7} \mathrm{yr}$ implies $\gamma \sim 5$. Their conclusion was based on the quasar luminosity function at $z \sim 3$. In this paper, we have found that the requirement of $\gamma \sim 5$ extends to the explanation of the space density of the highest redshift quasars known. Haenhelt, Natarayan, \& Rees (1998) suggested that the mass of central black holes may be regulated by feedback, which also implies $\gamma=5$. We return to discussion of this point in $\S 6$.
Ferrarese \& Merritt (2000) find a relation between $M_{\text {bh }}$ and bulge velocity dispersion $\sigma_{b}$ of $M_{\mathrm{bh}} \propto \sigma_{B}^{4.72 \pm 0.36}$, while Tremaine et al. (2002, and references therein) find $M_{\mathrm{bh}} \propto \sigma_{b}^{4.02 \pm 0.32}$. Tremaine et al. (2002) attribute this disagreement primarily to the differences in the velocity dispersion measurements used by the two groups. Unfortunately, the highly uncertain nonlinear scaling of $v_{c} \propto \sigma_{b}^{0.88 \pm 0.17}$ (Ferrarese 2002) allows the results of both groups to be consistent with our favored value of $\gamma \sim 5$ (which in turn translates to a mean slope of $M_{\mathrm{bh}} \propto \sigma_{b}^{4.4}$, in between the slopes of the two groups).

Due to its dependence on the exponential tail of the PressSchechter mass function, the model luminosity function is very sensitive to the value of $\sigma_{8}$. We have experimented by varying $\sigma_{8}$ after fixing $\epsilon=10^{-5.1}$ and $\gamma=4.71$ (local values from Ferrarese 2002). Values of $\sigma_{8}<0.7$ result in logarithmic slopes at $z \sim 4.3$ that are significantly steeper than observed, space densities at $z \sim 6$ that are smaller than observed, and duty cycles larger than $10^{7.5} \mathrm{yr}$. Similarly, values of $\sigma_{8}>1.0$ result in logarithmic slopes at $z \sim 4.3$ that are significantly shallower than observed, quasar space densities at $z \sim 6$ that are larger than observed, and duty cycles smaller than $10^{6.2} \mathrm{yr}$. Similar results are obtained if values of $\epsilon=10^{-5.4}$ and $\gamma=5$ are assumed. Thus, the high-redshift quasar luminosity function suggests $0.7 \lesssim \sigma_{8} \lesssim 1.0$.

The upper left hand panel of Figure 2 indicates that the model luminosity function provides a poorer fit to the data at redshifts $z \lesssim 2$. The density of relatively faint $\left(L_{B} \lesssim 10^{13} L_{\odot}\right.$ at $z \sim 1.0$ and $L_{B} \lesssim 10^{12} L_{\odot}$ at $z \sim 0.1$ [dashed lines]) quasars is reasonably reproduced, particularly at the lowest redshift considered. The model also predicts a decline in the number of bright quasars at low redshifts. On the other hand, the slope of the model luminosity function is too shallow at the higher luminosities to explain the data. A simple observational explanation for this phenomenon might be the large drop in the average cosmic density of cold galactic gas from $z \sim 2-3$ to the present day (StorrieLombardi, McMahon, \& Irwin 1996). Furthermore, the inability of the gas to cool following the epoch of group and $\mathrm{X}$-ray cluster formation would also contribute to the rapid decline in the luminosity function at low redshifts (Cavaliere 

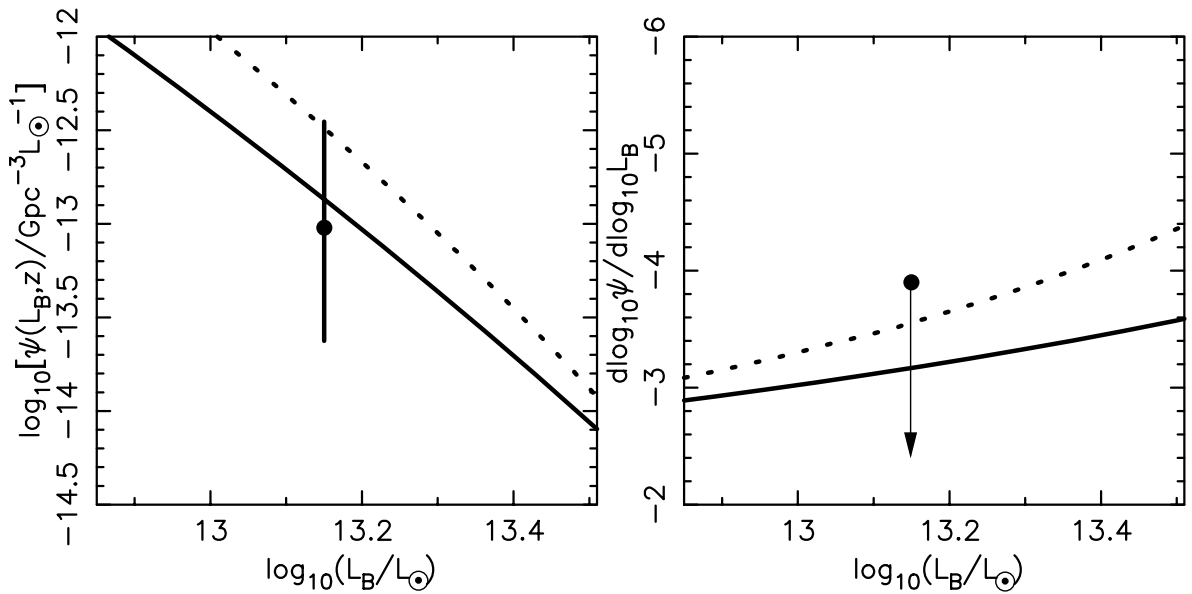

Fig. 5.-Detailed comparison of the observed rest-frame $B$-band quasar luminosity function with the models at $z \sim 6.0$. Left panel: Space density of quasars measured by Fan et al. (2001b). Solid and dotted lines: Luminosity functions for the halo merger model $\left(\epsilon_{0}=10^{-5.4}, \gamma=5, t_{\mathrm{dc}, 0}=10^{6.3} \mathrm{yr}\right)$ and for the HL98 model $\left(\epsilon=10^{-3.1}, t_{\mathrm{dc}, 0}=10^{5.0} \mathrm{yr}\right)$, respectively. Right panel: Logarithmic derivatives of the model luminosity functions. The empirical lower limit (95\%) is shown for comparison.

\& Vittorini 2000). Thus, while the merger rate predicts a decrease in the space density of quasars at low redshifts, the more complicated low-redshift environments require additional modeling (e.g., Kauffmann \& Haehnelt 2000) to reproduce the observed luminosity function shape.

\subsection{The Halo Formation Model}

We find that the HL98 model $(\S 2.1)$ requires $^{3}$ best-fit values of $\epsilon=10^{-3.1}$ and $t_{\mathrm{dc}, 0}=10^{5.0} \mathrm{yr}$. The resulting luminosity function is shown as the dotted lines in Figures 2 and 4. The HL98 model and the halo merger model produce similar luminosity functions at $z \gtrsim 2$. However, the predicted duty cycle is too low by 2 orders of magnitude and the value of $\epsilon$ produced by this fit is too high by 2 orders of magnitude to be consistent with observations.

Figures 4 and 5 indicate that the HL98 model produces a somewhat less consistent normalization at $z \sim 4.3$ and a steeper slope at $z \sim 6.0$ when compared with the halo merger model. At low redshifts, the merger rate is higher than the collapse rate for halos in the mass range of interest. In fact, the derivative of the Press-Schechter (1974) mass function becomes negative and thus no longer approximates the halo formation rate. At low redshifts, the model predicts a luminosity function with a sharp lower cutoff in luminosity [in the implementation of HL98 the smooth function, $f(t)$, results in a softer cutoff]. This is seen in the upper left panel of Figure 2.

\section{RATES OF MULTIPLE-IMAGE LENSING}

The fraction of quasars that are gravitationally lensed by foreground galaxies is very sensitive to the underlying luminosity function due to the potentially large magnification bias (Turner 1980; Turner, Ostriker, \& Gott 1984). At low redshifts $(z \lesssim 3)$, the bright end of the luminosity function has a universal slope of -3.43 . However, at $z \sim 4.3$, Fan et

\footnotetext{
${ }^{3}$ HL98 quote $t_{\mathrm{dc}, 0}=10^{5.8} \mathrm{yr}$, larger than our value of $t_{0}=10^{5}$. Haehnelt, Natarajan, \& Rees (1998) also found a lower value $t_{\mathrm{dc}, 0}$ in their model with constant $\epsilon$.
}

al. (2001a) observed a slope of -2.58 . Wyithe \& Loeb (2002a, 2002b) showed that empirical extrapolations of the low-redshift quasar luminosity function to high redshifts yielded very different results for the gravitational lensing rate if one were to adopt a bright end slope that is -3.43 at all redshifts or a slope that is -2.58 above $z \sim 3$. Since the halo merger model described in $\S 2.2$ reproduces all measured properties of the quasar luminosity function at high redshifts, we use it to compute the magnification bias for multiple-image gravitational lenses. The magnification bias can then be used to find the fraction of all quasars (at different redshifts) that are multiply imaged. The lensing fraction for the SDSS high-redshift samples was discussed at length in Wyithe \& Loeb (2002a, 2002b). We therefore give only a short summary here and present results for the lens fraction obtained with the new luminosity function.

The probability distribution of point-source magnification by a singular, spherical isothermal lens in terms of the sum of the magnifications of multiple images, $\mu$, is $(d P / d \mu)=8 / \mu^{3}$ for $\mu>2$. The bias factor for multiple imaging in a flux-limited sample brighter than (absolute luminosity) $L_{\mathrm{B}, \lim }$ is therefore

$$
B(z)=\frac{\int_{2}^{\infty} d \mu\left[8 /\left(\mu^{3}\right)\right] \int_{L_{B, \text { lim }} / \mu}^{\infty} d L^{\prime} \Psi\left(L^{\prime}, z\right)}{\int_{L_{B, \text { lim }}}^{\infty} d L^{\prime} \Psi\left(L^{\prime}, z\right)} .
$$

This magnification bias is plotted as a function of the limiting (absolute) luminosity in Figure 6. Biases at redshifts of $z=2.3, z=4.3$, and $z=6.0$ are shown as well as predictions for $z \sim 8.0$ and $z \sim 10.0$ (bottom to top). The bias becomes very large at high redshifts and for bright limiting magnitudes.

The resulting fraction of multiple images expected in the flux-limited sample is

$$
F\left(L_{B, \lim }, z\right) \approx \frac{B \tau_{\text {mult }}}{B \tau_{\text {mult }}+\left(1-\tau_{\text {mult }}\right)} .
$$

In this expression, $\tau_{\text {mult }}$ is the multiple image optical depth, i.e., the source plane probability that a source will be multiply imaged for the observer. From Wyithe \& Loeb (2002b), we find $\tau_{\text {mult }}=0.0040$ for the SDSS quasars at $z \sim 4.3$ and 

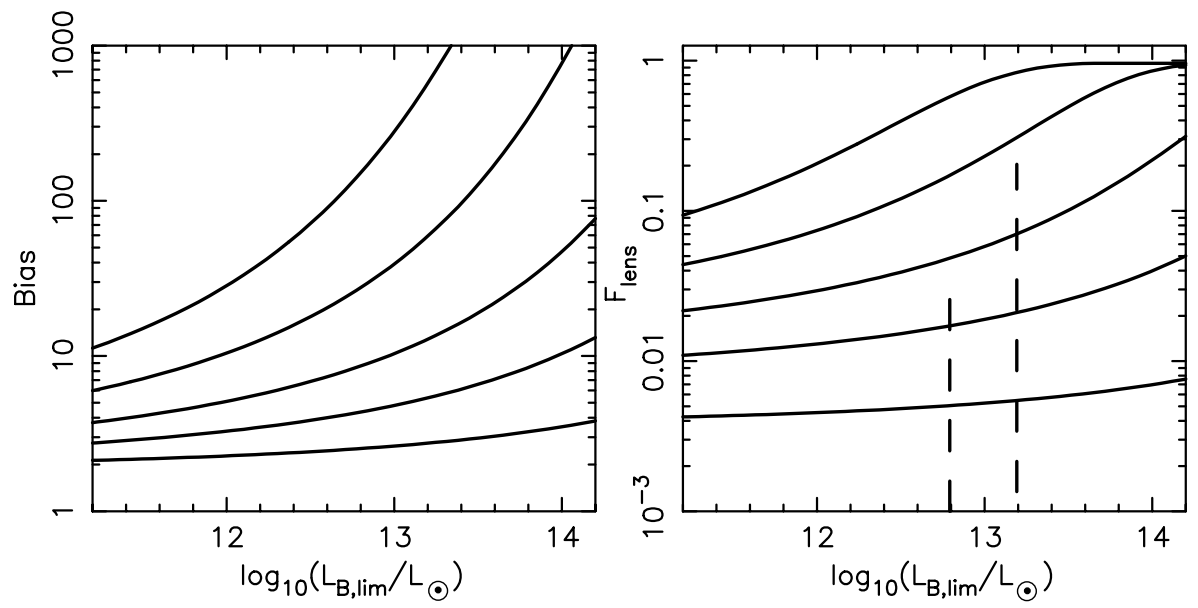

FIG. 6.- Left: Magnification bias as a function of absolute luminosity limit for flux limited samples of quasars at $z=2.3,4.3,6.0,8.0$, and 10.0 (bottom to top). Right: Multiple-image lensing rate for flux limited samples of quasars at $z=2.3,4.3,6.0,8.0$, and 10.0 (bottom to top). Left and right vertical dashed lines: Absolute flux limit of the SDSS samples at $z \sim 4.3$ and $z \sim 6.0$, respectively.

$\tau_{\text {mult }}=0.0059$ for the SDSS quasars at $z \sim 6$. In addition, we find $\tau_{\text {mult }}=0.0020, \tau_{\text {mult }}=0.0076$, and $\tau_{\text {mult }}=0.0091$ (considering all galaxies as potential lenses) at $z=2.3$, $z=8.0$, and $z=10.0$, respectively. The lens fraction $F\left(L_{B, \lim , z}\right)$ is plotted in the right-hand panel of Figure 6 for $z=2.3,4.3,6.0,8.0$, and 10.0 (bottom to top). While at low redshifts the lensing fraction is less than $1 \%$, at higher redshifts, the fraction could be much higher. The vertical dashed lines correspond to the limiting absolute luminosities of the SDSS quasar samples at $z \sim 4.3$ (left) and $z \sim 6$ (right). These limits suggest lens fractions of $\sim 2 \%$ in the SDSS sample at $z \sim 4.3$ and $\sim 8 \%$ in the SDSS sample at $z \gtrsim 5.7$. Note that the fractions at $z \sim 4.3$ and $z \sim 6.0$ are consistent with the value obtained for the flat luminosity function assumed in Wyithe \& Loeb (2002b). At even higher redshifts, the lens fraction approaches unity. The luminosity function at $z \sim 10$ predicts that quasars having luminosities brighter than a limit that corresponds to the same comoving space density as the SDSS quasars at $z \sim 6.0$ have a lens fraction of $F \sim 0.5$.

\section{SUMMARY AND DISCUSSION}

We predict the quasar luminosity function under the hypothesis that quasar activity is associated with galaxy mergers. Galactic halos are assumed to each host a black hole having a mass determined from a power-law relation with halo circular velocity, $M_{\mathrm{bh}} \propto v_{c}^{\gamma}$. Upon merger, we assume that the gas from the accreted halo is driven to the center and that the quasar then shines at its Eddington rate for a short period of time, proportional to the (smaller than one-half) mass ratio between the small and final halos in the merger. Our model has three free parameters but at least the following five constraints: the luminosity function slope and normalization at $z \sim 2.5$, the evolutions of the luminosity function slope and normalization, and the curvature of the luminosity function. Our model is simplified by the fact that the effect of reionization on the luminosity function is limited to luminosities much fainter than those detectable in current quasar surveys.
This very simple model reproduces all known properties of the luminosity function above $z \sim 2$, including the recently measured luminosity function slope at $z \sim 4.3$ (Fan et al. 2001a) and the space density of quasars at $z \sim 6.0$ (Fan et al. 2001b). The model suggests a black hole-halo mass relation $M_{\mathrm{bh}} \propto v_{c}^{5}$, consistent with that found by Ferrarese (2002) at $z=0$, and a duty cycle of $\sim 10^{7} \mathrm{yr}$, consistent with previous determinations ( $\mathrm{Yu} \&$ Tremaine 2002; Steidel et al. 2002). An acceptable fit to all of these constraints requires $0.7 \lesssim \sigma_{8} \lesssim 1.0$. Using the derived luminosity function, we predict the resulting lensing rates for the highest redshift samples. The recently published flux-limited samples of SDSS quasars at $z \sim 4.3$ and $z \gtrsim 5.7$ are predicted to have multiple image fractions of $\sim 2 \%$ and $\sim 8 \%$, respectively.

The model luminosity function computed using the halo merger rates is consistent with the density of faint quasars at low redshifts and predicts a decline in the density of bright quasars toward low redshifts but predicts a luminosity function slope that is shallower than observed. As has been noted previously, a more careful analysis (e.g., Kauffmann $\&$ Haehnelt 2000) is required to understand the evolution of the quasar luminosity function at low redshifts.

The $M_{\mathrm{bh}}-v_{c}$ relation that provides the best fit to the data may also have a simple physical origin. First, consider dimensional analysis. If we make the minimal assumption that the limiting luminosity of a quasar is only a function of the halo circular velocity $v_{c}$, then the only dimensional parameters in the problem are $v_{c}$ and $G$ (Newton's constant). The only combination of these parameters that has dimensions of luminosity is $v_{c}^{5} / G$, and so we conclude

$$
L_{\mathrm{Edd}} \propto \frac{v_{c}^{5}}{G}
$$

What is the physics behind this relation? The value of $v_{c}^{5} / G$ amounts to depositing the entire binding energy of a selfgravitating system during its dynamical time (so that it does not have time to adjust). The binding energy of a self-gravitating mass $M$ is $\sim M v_{c}^{2}$, and the dynamical time is $\sim r / v_{c}$. The ratio between these quantities is $\sim M v_{c}^{3} / r$. Using the virial relation $G M / r \sim v_{c}^{2}$, we get the energy deposition rate 
that would unbind a self-gravitating system on its dynamical time, $\sim v_{c}^{5} / G$. A quasar may therefore unbind the gas in the galaxy around it ${ }^{4}$ if its power output is too large ${ }^{5}$ (Silk \& Rees 1998; Ciotti \& Ostriker 2001). As the mass of the black hole increases and the quasar's Eddington luminosity approaches this limit, the feedback will generate a powerful galactic wind and terminate the accretion that feeds the quasar. Hence, the quasar phenomenon may be self-terminating (similarly to the formation of a protostar). Of course, if some of the quasar energy escapes (due to incomplete absorption, partial covering factor, or efficient cooling by the surrounding gas), then the feedback-limited quasar luminosity would be higher than $v_{c}^{5} / G$. As long as the feedback from all quasars encounters self-similar conditions in different halos, we may write

$$
L_{\mathrm{Edd}}=\beta_{\epsilon} \frac{v_{c}^{5}}{G},
$$

\footnotetext{
${ }^{4}$ We implicitly assume that the central region of the galaxy (such as the protobulge) is characterized by a circular velocity similar to that of the galactic halo, even though it is dominated by baryons. This assumption follows naturally from the nearly flat rotation curves of nearby galaxies.

${ }^{5}$ Note that the power output from the quasar may include both radiation and mechanical energy. Substantial outflows are inferred to exist in broad absorption line quasars and radio galaxies (see recent summary in Furlanetto \& Loeb 2001 and references therein)
}

where $\beta_{\epsilon}$ is a constant that is related to the inferred value of $\epsilon_{0}$. Equations (10), (11), and (24) and the best-fit value of $\epsilon_{0} \sim 10^{-5.4}$, together with the relation $L_{\mathrm{Edd}}=1.4 \times 10^{38}\left(M_{\mathrm{bh}} / M_{\odot}\right) \mathrm{ergs} \mathrm{s}^{-1}$, yield $\beta_{\epsilon} \approx 30$. We have shown that the assumption of a constant $\beta_{\epsilon}$ in equation (24) leads to a luminosity function that describes the data over a wide range of redshifts. The constancy of $\beta_{\epsilon}$ (which is also expected from dimensional analysis) implies that feedback may indeed be the mechanism that regulates the growth of supermassive black holes in galactic potential wells. The similarity between our deduced values of $\gamma=5$ and $\epsilon_{0}=10^{-5.4}$ at high redshifts and the values inferred for remnant black holes in the local universe (Ferrarese 2002; Tremaine et al. 2002) provides a strong testimony to this effect.

We thank the anonymous referee for several suggestions that improved the results of the paper. This work was supported in part by NSF grants AST-9900877 and AST0071019 (for A. L). J. S. B. W. is supported by a Hubble Fellowship grant from the Space Telescope Science Institute, which is operated by the Association of Universities for Research in Astronomy, Inc., under NASA contract NAS 5-26555.
Bardeen, J. M., Bond, J. R., Kaiser, N., \& Szalay, A. S. 1986, ApJ, 304, 15 Barkana, R., \& Loeb, A. 2001, Phys. Rep., 349, 125

Bond, J. R., Cole, S., Efstathiou, G., \& Kaiser, N. 1991, ApJ, 379, 440

Boyle, B. J., Shanks, T., Croom, S. M., Smith, R. J., Miller, L., Loaring, N., \& Heymans, C. 2000, MNRAS, 317, 1014

Boyle, B. J., Shanks, T., \& Peterson, B. A. 1988, MNRAS, 235, 935

Carlberg, R. G. 1990, ApJ, 350, 505

Cavaliere, A., \& Vittorini, V. 2000, ApJ, 543, 599

Ciotti, L., \& Ostriker, J. P. 2001, ApJ, 551, 131

Efstathiou, G., \& Rees, M. J. 1988, MNRAS, 230, 5

Elvis, M., et al. 1994, ApJS, 95, 1

Fan, X., et al. 2001a, AJ, 121, 54 . $2001 \mathrm{~b}, \mathrm{AJ}, 122,2833$

Ferrarese, L. 2002, ApJ, 578, 90

Ferrarese, L., \& Merritt, D. 2000, ApJ, 539, L9

Fukugita, M., Ichikawa, T., Gunn, J. E., Doi, M., Shimasaku, K., \& Schneider, D. P.. 1996, AJ, 111, 1748

Furlanetto, S. R., \& Loeb, A. 2001, ApJ, 556, 619

Gunn, J. E et al. 1998, AJ, 116, 3040

Haehneit, M. G., Natarajan, P., \& Rees, M. J. 1998, MNRAS, 300, 817

Haehnelt, M. G., \& Rees, M. J. 1993, MNRAS, 263, 168

Haiman, Z. \& Loeb, A. 1998, ApJ, 503, 505 (HL98)

Haiman, Z., Madau, P., \& Loeb, A. 1998, ApJ, 514, 535

Hartwick, F. D. A., \& Schade, D. 1990, ARA\&A, 28, 437

Hernquist, L., \& Mihos, J. C. 1995, ApJ, 448, 41

Kauffmann, G., \& Haehnelt, M. 2000, MNRAS, 311, 576

\section{EFERENCES}

Kauffmann, G., \& Haehnelt, M. 2002, MNRAS, 332, 529

Kennefick, J. D., Djorgovski, S. G., \& de Carvalho, R. R. 1995, AJ, 110 , 2553

Lacey, C., \& Cole, S. 1993, MNRAS, 262, 627

Medvigy, D., \& Loeb, A. 2002, ApJ, submitted (astro-ph/0110014)

Mihos, J. C., \& Hernquist, L. 1994, ApJ, 431, L9

Pei, Y. C. 1995, ApJ, 438, 623

Press, W. H., \& Schechter, P. 1974, ApJ, 187, 425

Schmidt, M., Schneider, D. P., \& Gunn, J. E. 1995, AJ, 110, 68

Schneider, D. P. et al. 2001, AJ, 121, 1232

Sheth, R. K. \& Tormen, G. 1999, MNRAS, 308, 119

Silk, J., \& Rees, M. J. 1998, A\&A, 331, L1

Small, T. A., \& Blandford, R. D. 1992, MNRAS, 259, 725

Steidel, C. C., Hunt, M. P., Shapley, A. E., Adelberger, K. L., Pettini, M., Dickinson, M., \& Giavalisco, M. 2002, ApJ, 576, 653

Storrie-Lombardi, L. J., MacMahon, R. G., \& Irwin, M. J. 1996, MNRAS, 283, 79

Tremaine, S., et al. 2002, preprint (astro-ph/0203468)

Turner, E. L. 1980, ApJ, 242, L135

Turner, E. L., Ostriker, J. P., \& Gott, J. R., III. 1984, ApJ, 284, 1

Warren, S. J., Hewett, P. C., \& Osmer, P. S. 1994, ApJ, 421, 412

Wyithe, J. S. B., \& Loeb, A. 2002a, Nature, 417, 923 2002b, ApJ, 577, 57

York, D. G., et al. 2000, AJ, 120, 1579

Yu, Q., \& Tremaine, S. 2002, MNRAS, 335, 965 